\title{
SO(6)-Generalized Pseudogap Model of the Cuprates
}

\author{
R.S. Markiewicz ${ }^{1,2}$, C. Kusko ${ }^{1,2, *}$, and M.T. Vaughn ${ }^{1}$ \\ Physics Department (1) and Barnett Institute (2), Northeastern U., Boston MA 02115
}

\begin{abstract}
The smooth evolution of the tunneling gap of $\mathrm{Bi}_{2} \mathrm{Sr}_{2} \mathrm{CaCu}_{2} \mathrm{O}_{8}$ with doping from a pseudogap state in the underdoped cuprates to a superconducting state at optimal and overdoping reflects an underlying $\mathrm{SO}(6)$ instability structure of the $(\pi, 0)$ saddle points. The pseudogap is probably not associated with superconductivity, but is related to competing nesting instabilities, which are responsible for the stripe phases.

We earlier introduced a simple Ansatz of this competition in terms of a pinned Balseiro-Falicov (pBF) model of competing charge density wave and (s-wave) superconductivity. This model gives a good description of the phase diagram and the tunneling and photoemission spectra. Here, we briefly review these results, and discuss some recent developments: experimental evidence for a non-superconducting component to the pseudogap; and $\mathrm{SO}(6)$ generalizations of the pBF model, including flux phase and d-wave superconductivity.
\end{abstract}

Recent photoemission [1,2] and tunneling [3,4] studies in underdoped cuprates find a remarkably smooth evolution of the pseudogap into the superconducting gap as doping increases. This has led to the suggestion that the pseudogap is caused by superconducting fluctuations or precursor pairing [5]. We suggest alternatively that the pseudogap represents a competing ordered state closely related to the stripe phases, with the smooth evolution due to an underlying $\mathrm{SO}(6)$ symmetry of the instabilities of the Van Hove singularity (VHS).

In this picture, the stripe phases represent a nanoscale phase separation, between a magnetic (spin-density wave or flux phase) instability at half filling and a chargedensity wave (CDW) near optimal doping [6]. We have introduced a simple Ansatz, the pinned Balseiro-Falicov (pBF) [7,8] model, which captures the essential features of the stripe-superconductivity competition.

Pseudogap Phase Diagram: By comparing simultaneous measurements [9] of the photoemission gap $\Delta$ with the pseudogap onset temperature $T^{*}$, we find an approximately constant ratio $2 \Delta(0) / k_{B} T^{*} \simeq 8$, which allows us to plot the Bi-2212 pseudogap phase diagram as $T^{*}$ vs $x$, providing a direct comparison with transport-derived pseudogaps in LSCO and YBCO [10], Fig. 1a. Remarkably, all three materials scale onto a single, universal phase diagram, the scaling involving 

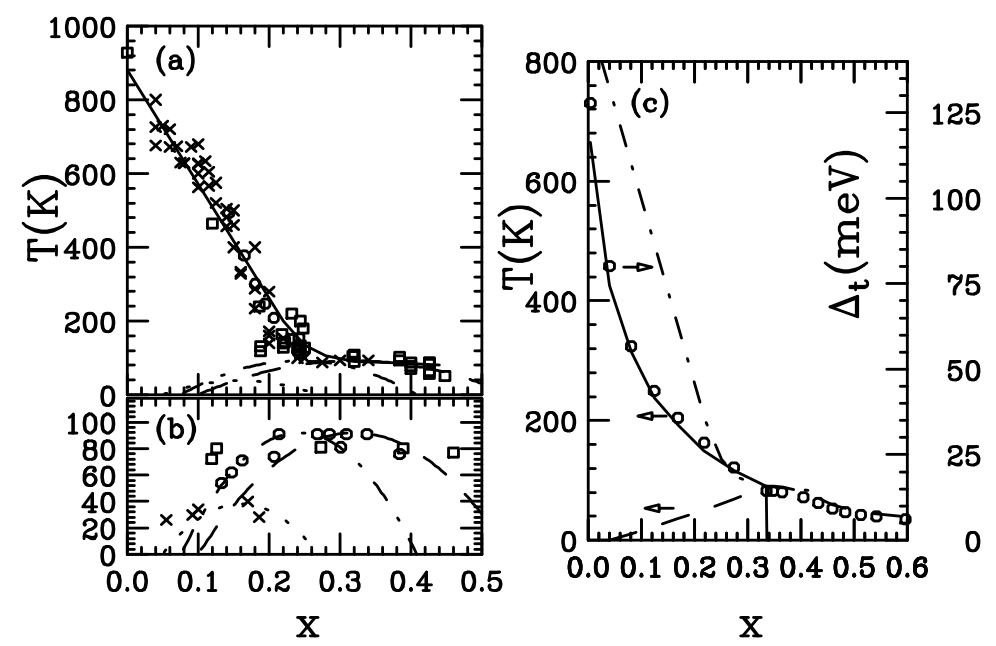

FIGURE 1. (a) Pseudogap phase diagram for Bi-2212 (squares) determined from photoemission [9], and LSCO ( $\times$ 's) and YBCO (circles) determined from transport [10]. (YBCO data shifted by $10 \%$ for better scaling.) Solid line = guide to the eyes; other lines $=$ parabolic approximations to superconducting $T_{c}$ for LSCO (dotted line), YBCO (dashed line) and Bi-2212 (dot-dashed line). (b) Uemura plot, with $n / m$ scaled to $x$. Symbols and curves have same meanings as in (a). (c) Model pseudogap phase diagram for Bi-2212. Solid line $=\mathrm{CDW}$ transition $T_{p}$; dashed line $=$ superconducting transition $T_{c}$; circles $=$ total gap $\Delta_{t}$ at $1 \mathrm{~K}$; dotdashed line $=$ solid line from Fig.1a.

only a shift of the x-axes, relative to LSCO. Such a shift is, however, not consistent with a universal scaling of the superconducting $T_{c}$ 's - optimal $\mathrm{T}_{c}$ falls at a different $x$ for each material (parabolic curves in Fig. 1a,b). On the other hand, the Uemura plots [11] also find that optimal $T_{c}$ falls at very different values of $n / m$ for different cuprates. The simple assumption [12] that $n / m \propto x$ (with the constant of proportionality fixed by the LSCO data), unifies the Uemura plot (symbols in Fig. 1b) with the pseudogap scaling of $T_{c}$ (curves, Fig. 1b). This strongly suggests that the scaling for YBCO and Bi-2212 merely converts the data to the correct value of $\mathrm{x}$. The resulting phase diagram can be well fit by the pBF model, Figure 1c, although the ratio $2 \Delta / k_{B} T^{*}$ is 4.1 , somewhat lower than experiment.

SO(6): The group structure of the model should be thought of not as a symmetry group, but more in a renormalization group sense, as in the one-dimensional metal g-ology. (The group structure of g-ology has been discussed by Solomon and Birman [13].) Due to the logarithmic divergence of the density of states near a VHS, the Fermi surface almost reduces to two points - the VHS's at $(\pi, 0)$ and $(0, \pi)$. The possible instabilities of the model have an underlying $\mathrm{SO}(6)$ symmetry [14], but which instabilities are observed depend sensitively on the form of the coupling constants - corresponding to the g's of g-ology. There are fundamentally two classes of instability - nesting instabilities which couple the two VHS's and pairing instabilities, which are intra-VHS. 


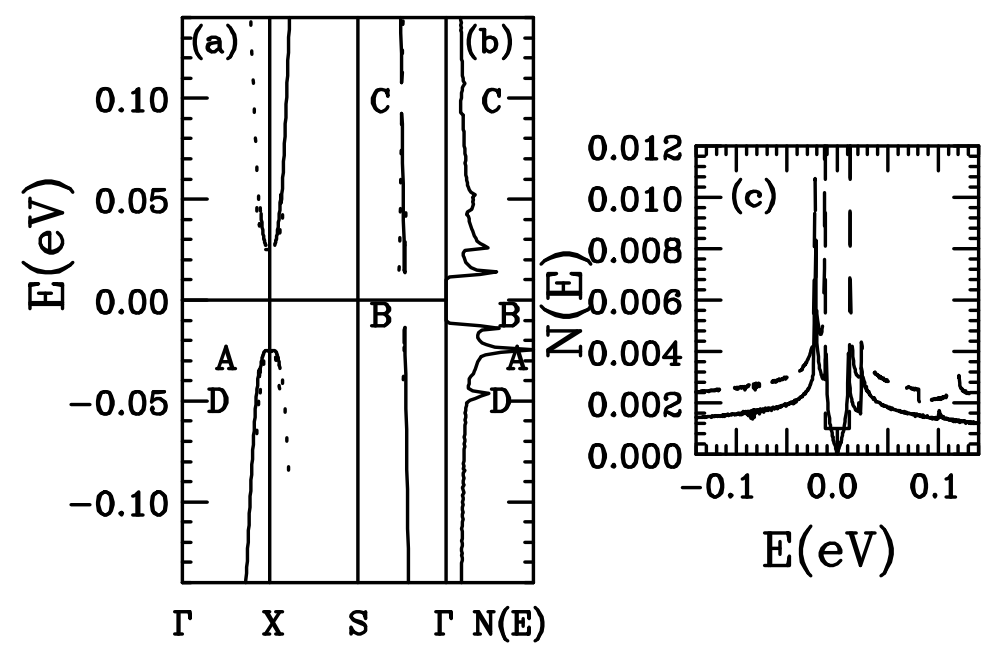

FIGURE 2. (a) Energy dispersion near Fermi level for underdoped cuprate [7], illustrating spectral weight. Coherence factor $\geq 0.6$ : solid lines; between 0.1 and 0.6 : dashed lines; between 0.001 and 0.1: dotted lines. (b) Tunneling dos. (c) Comparing tunneling dos for CDW-s-wave superconductivity (dashed line, shifted up by 0.001) and flux phase - d-wave superconductivity (solid line).

The $\mathrm{SO}(6)$ symmetry of the model is most clearly manifested in the equation for the total gap at $(\pi, 0)$ :

$$
\Delta_{t}=\sqrt{\sum_{i} \Delta_{i}^{2}},
$$

where the $\Delta_{i}$ are the individual gaps associated with each instability. (Note the g-ology flavor of this result: there is no underlying symmetry which says that all the $\Delta_{i}$ 's are equal.) In this case, the pBF model amounts to the replacement $\Delta_{S D W}^{2}+\Delta_{C D W}^{2} \rightarrow \Delta_{p}^{2}$, where $\Delta_{p}$ is the net pseudogap, which has similar form to a CDW gap. Equation 1 shows that the smooth evolution of the tunneling gap with doping is consistent with a crossover from magnetic behavior near half filling to superconducting behavior at optimal doping.

Photoemission and Tunneling Spectra: Fig. 2 compares the energy dispersion (a) and the tunneling spectra (b) near the Fermi level, in the underdoped regime. It can be seen that structure in the tunneling dos is directly related to features in the dispersion of the gapped bands. Thus, peak A is associated with the dispersion at $(\pi, 0)$ - the VHS peak split by the combined CDW-superconducting gap. Peak B is due to the superconducting gap away from $(\pi, 0)$ - particularly near $(\pi / 2, \pi / 2)$. Feature $\mathrm{C}$ is associated with the CDW gap $G_{k}$ near $(\pi / 2, \pi / 2)$.

An equation similar to Eq. 1 arises in the theory of Bilbro and McMillan [15] - also a (three-dimensional) VHS theory - and was postulated to explain thermodynamic data on the pseudogap [16]. What is new here is that the vector addition is found to hold only near the saddle points, while the gaps split near $(\pi / 2, \pi / 2)$, and only 


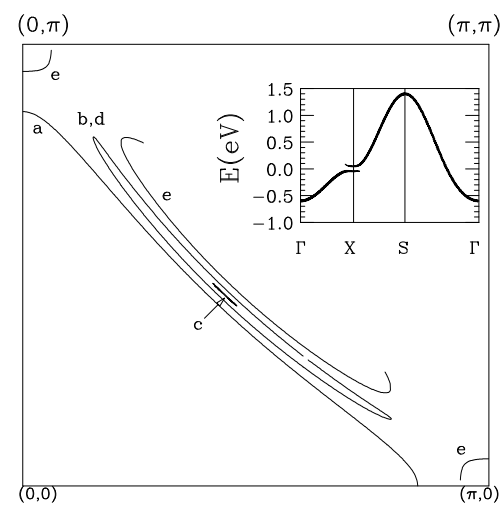

FIGURE 3. Constant energy surfaces for mixed flux phase - d-wave superconductivity: energy $=-50$ (a), -20 (b), -1 (c), 20 (d), and $50 \mathrm{meV}$ (e). Inset: Corresopnding dispersion, with thickness of line proportional to coherence factor.

the superconducting gap is near the Fermi surface there. This is consistent with Panagopoulos and Xiang [17], who found that, near the gap zero at $(\pi / 2, \pi / 2)$, the slope of the gap scales with $T_{c}$, and not with the gap near $(\pi, 0)$. Similarly, Mourachkine [18] has found evidence for two tunneling gaps, very similar to features $\mathrm{A}$ and $\mathrm{B}$ of Fig. 2b; as predicted, feature $\mathrm{B}$ scales with $\mathrm{T}_{c}$, and not with the pseudogap, feature A. Feature B arises from the superconducting gap at the hole pockets, as can be seen from the energy dispersion at the B gap energy, curve b,d in Fig. 3.

The phase diagram is most naturally fit by assuming that the pseudogap represents a nesting instability, and superconductivity a pairing instability. A more precise determination will require careful experimentation. Thus, Fig. 2c compares the tunneling spectra for two models, the original pBF model in terms of a CDW and an s-wave superconductor, and a modified version involving flux phase - $\mathrm{d}$-wave superconductivity competition. The resulting spectra are, as expected, nearly identical. Close inspection shows differences near $(\pi, \pi)$, where the gap is purely due to the pairing instability.

Van Hove Pinning: An essential ingrediant of the model is that the gap remains centered at $(\pi, 0)$ over the full doping range from half filling to optimal doping - that is, that the VHS is pinned at the Fermi level. This remarkable consequence of strong correlation effects was first pointed out in 1989 [19], and has been rederived numerous times since then [20]. We have noted that this pinning should be measurable, both in tunneling and in photoemission, and a preliminary analysis of the data appears to confirm the prediction [7].

Evidence for a Nonsuperconducting Pseudogap: The photoemission and tunneling spectra near optimal doping have a very characteristic form below $T_{c}$. There is a sharp quasiparticle peak at an energy $\Delta$ above (or below) the Fermi level, with a pronounced dip near $2 \Delta$, followed by a broad hump at higher energies. The dip is most probably associated with reduced quasiparticle scattering within 


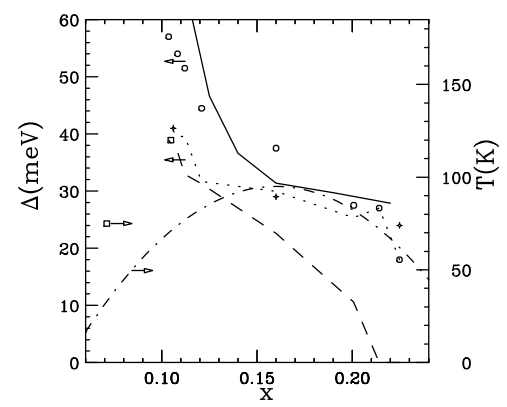

FIGURE 4. Tunneling gaps in BSCCO. Circles = net tunneling gap, $\Delta ;$ dotted (dashed) line $=$ estimate for $\Delta_{s}\left(\Delta_{p}\right)$; dotdashed line $=T_{c} ;{ }^{\prime}$ 's $=10 I_{c} R$, where $I_{c} R$ is the average $I_{c} R$ product [22]; squares $=$ estimate of $\mathrm{T}_{c 0}$ from Ref. [24]; solid line: from Ref. [7].

the superconducting state, which terminates when pairbreaking sets in at energies above twice the superconducting gap, $2 \Delta_{s}$ [21]. Recently, Miyakawa, et al. [22] showed how the tunneling gap in Bi-2212 evolves with doping, scaling a series of tunneling curves to the respective $\Delta$ 's. These curves show significant deviations from scaling of the dip feature with the tunneling gap, which suggest that in the underdoped regime, $\Delta_{s}<\Delta$. By assuming that the dip scales exactly with $\Delta_{s}$, it is possible to extract the doping dependence of $\Delta_{s}$, and correspondingly of $\Delta_{p}$, the non-superconducting component of the gap [23], Fig. 4.

Shown also is recent Terahertz data from Corson, et al. [24], who extract a bare superconducting transition temperature $T_{c 0}>T_{c}$ from a Berezinski-KosterlitzThouless analysis of superconducting fluctuations. (The effective $T_{c 0}$ is found to be strongly frequency dependent; the squares in Fig. 4 are an estimate based on the highest frequency data, 600GHz.) Note that $T_{c 0}$ is considerably smaller than the pseudogap onset and has very different scaling, actually decreasing with increased underdoping. Indeed, this $T_{c 0}$ is consistent with the values of $\Delta_{s}$ estimated in Ref. [23], with the same ratio of $\Delta$ to $T_{c}$ as found for the total gap in the overdoped regime (where the nonsuperconducting component is absent). The data suggest a rather modest pair-breaking effect of the stripes, reducing the optimal $T_{c}$ from $\sim 125 K$ to $95 K$.

Conclusions: The simple pinned Van Hove Ansatz for the striped pseudogap phase in the cuprates provides a detailed explanation for the phase diagram and the experimental tunneling and photoemission spectra. In particular: (1) The fact that the tunneling peaks are experimentally found to coincide with the $(\pi, 0)$ photoemission dispersion [4] shows that the $(\pi, 0)$ dispersion has a gap - that is, that the pseudogap is associated with VHS nesting [6]. (2) The interpretation is selfconsistent, in that the experiments seem to find that the Fermi level is pinned near the VHS in the underdoped regime [7]. (3) The tunneling gap has a characteristic asymmetry which vanishes at optimal doping; this is evidence that optimal doping is that point at which the Fermi level exactly coincides with the VHS [7]. (4) While there are superconducting fluctuations in the underdoped regime, a large 
fraction of the pseudogap has a non-superconducting origin. (5) Portions of the tunneling spectra associated with the Fermi surface near $(\pi / 2, \pi / 2)$ show distinct scaling with $T_{c}$, not $T^{*}$. (6) The pseudogap phase diagrams for Bi-2212, LSCO, and YBCO appear to be universal and consistent with the Uemura plot, while optimal doping $x_{c}$ varies from compound to compound.

Our interest in the tunneling studies was sparked by conversations with A.M. Gabovich. We would like to thank NATO for enabling him to visit us. MTV's work was supported by DOE Grant DE-FG02-85ER40233. Publication 757 of the Barnett Institute.

* : On leave of absence from Inst. of Atomic Physics, Bucharest, Romania

\section{REFERENCES}

1. D.S. Marshall, et al., Phys. Rev. Lett. 76, 4841 (1996); A.G. Loeser, et al., Science 273, 325 (1996).

2. H. Ding, et al., Nature 382, 51 (1996).

3. Ch. Renner, et al., Phys. Rev. Lett. 80, 149 (1998).

4. N. Miyakawa, et al., Phys. Rev. Lett. 80, 157 (1998).

5. M. Randeria, unpublished (cond-mat/9710223).

6. R.S. Markiewicz, Phys. Rev. B56, 9091 (1997).

7. R.S. Markiewicz, C. Kusko and V. Kidambi, unpublished (cond-mat/9807068).

8. C. Balseiro and L. Falicov, Phys. Rev. B20, 4457 (1979).

9. H. Ding, et al., J. Phys. Chem. Sol. 59, 1888 (1998).

10. B. Batlogg, et al., Physica C235-240, 130 (1994).

11. Y.J. Uemura, et al., Phys. Rev. Lett. 62, 2317 (1989).

12. R.S. Markiewicz and B.C. Giessen, Physica C160, 497 (1989).

13. A.I. Solomon and J.L. Birman, J. Math. Phys. 28, 1526 (1987).

14. R.S. Markiewicz and M.T. Vaughn, J. Phys. Chem. Sol. 59, 1737 (1998), and Phys. Rev. B57, 14052 (1998).

15. G. Bilbro and W.L. McMillan, Phys. Rev. B14, 1887 (1976).

16. J.W. Loram, et al., J. Supercond. 7, 243 (1994).

17. C. Panagopoulos and T. Xiang, Phys. Rev. Lett. 81, 2336 (1998).

18. A. Mourachkine, unpublished (cond-mat/9810161).

19. R.S. Markiewicz, J. Phys. Cond. Matt. 2, 665 (1990).

20. Earlier references summarized on p. 1223 of R.S. Markiewicz, J. Phys. Chem. Sol. 58, 1179 (1997); more recent references include N. Furukawa and T.M. Rice, J. Phys. Cond. Matt. 10, L381 (1998), and G. Hildebrand, E. Arrigoni, C. Gröber, and W. Hanke, unpublished (cond-mat/9801181).

21. D. Coffey and L. Coffey, Phys. Rev. Lett. 70, 1529 (1993).

22. N. Miyakawa, et al., unpublished (cond-mat/9809398).

23. R.S. Markiewicz and C. Kusko, unpublished (cond-mat/9810214).

24. J. Corson, et al., unpublished (cond-mat/9810280). 